\title{
Joint imputation of whole-genome sequence variants and large chromosomal deletions in cattle
}

\author{
Md Mesbah-Uddin, ${ }^{1,2 *} \odot$ Bernt Guldbrandtsen, ${ }^{1} \odot$ Mogens Sandø Lund, ${ }^{1}$ Didier Boichard, ${ }^{2} \odot$ \\ and Goutam Sahana ${ }^{1 *}$ (i) \\ ${ }^{1}$ Department of Molecular Biology and Genetics, Center for Quantitative Genetics and Genomics, Aarhus University, 8830 Tjele, Denmark \\ ${ }^{2} \mathrm{GABI}$, INRA, AgroParisTech, Université Paris-Saclay, 78350 Jouy-en-Josas, France
}

\section{ABSTRACT}

Genotype imputation, often focused on SNP and small insertions and deletions (indels; size $\leq 50 \mathrm{bp}$ ), is a crucial step for association mapping and estimation of genomic breeding values. Here, we present strategies to impute genotypes for large chromosomal deletions (size >50 bp), along with SNP and indels in cattle. The pipelines include a strategy for extending the wholegenome sequence reference panel for large deletions, a 2-step genotype refinement approach using Beagle4 and SHAPEIT2 software, and finally, joint imputation of SNP, indels, and large deletions to the existing SNP array-typed population using Minimac3 software. Using these pipelines we achieved an imputation accuracy of the squared Pearson correlation $\left(\mathrm{r}^{2}\right)>0.6$ at minor allele frequencies as low as $0.7 \%$ for SNP and indels, and $0.2 \%$ for large deletions. This highlights the potential of our approach to build a haplotype reference panel and impute different classes of sequence variants across a wide allele frequency spectrum with high accuracy. Key words: Gaussian mixture model, imputation, structural variant, copy-number variation, chromosomal deletion

\section{INTRODUCTION}

Whole-genome resequencing in different cattle breeds has generated a comprehensive catalog of small sequence variants (size $\leq 50 \mathrm{bp}$ ) such as SNP and insertions and deletions (indels; Daetwyler et al., 2014). Structural variants (SV) are another class of DNA polymorphism that affect a large (usually $>50 \mathrm{bp}$ ) portion of the genome, which include deletions, insertions, duplications, inversions, and translocations (Alkan et al., 2011). The $\mathrm{SV}$ include both balanced and unbalanced sequence

Received May 10, 2019.

Accepted August 25, 2019.

*Corresponding authors: mdmesbah@gmail.com and goutam. sahana@mbg.au.dk rearrangements. Unbalanced SV, that is, the occurrences of fewer (deletions) or more copies (duplications) of some sequences in an organism compared with the reference genome, are known as copy number variants (CNV; Alkan et al., 2011). In cattle, whole-genome sequences (WGS) were used (though less frequently) to identify SV (Boussaha et al., 2015; Chen et al., 2017) and CNV (Letaief et al., 2017).

Interest is growing in understanding the phenotypic effects of SV (e.g., large deletions and duplications) in livestock because a substantial proportion of SV overlap genes and may have a biological effect (Bickhart and Liu, 2014). For example, in Holstein cattle, a 138-kb deletion on chromosome 9 (located between positions 93,232,654 and 93,370,998 bp; OMIA ID: 001941-9913) causes abortion (Schutz et al., 2016), whereas a 3.3-kb deletion on chromosome 21 (located between positions 21,184,869 and 21,188,202 bp; OMIA ID: 000151-9913) is responsible for fetal death and brachyspina (Charlier et al., 2012). Similarly, in Nordic Red Dairy cattle (RDC), a 662-kb deletion on chromosome 12 (located between positions 20,100,648 and 20,763,119 bp; OMIA ID: 001901-9913) causes embryonic mortality (Kadri et al., 2014), whereas a 525-kb deletion on chromosome 23 (located between positions 12,291,761 and 12,817,087 bp; OMIA ID: 001991-9913) is a causal factor for stillbirth (Sahana et al., 2016). Mapping of such a genomic region requires availability of SV genotypes in large cohorts of animals, which is rarely the case. One way to generate such SV data sets is to design a customized SNP array using the precise SV breakpoints and genotype the population directly. The custom part of the EuroG10K BeadChip (Boichard et al., 2018) is an example of this. Alternatively, one can perform imputation for common biallelic SV in an approach similar to that of biallelic SNP and indels because common SV are often tagged well by the surrounding SNP haplotypes [as shown by studies in humans, e.g., Conrad et al. (2010) and Handsaker et al. (2015)]. The latter approach will allow us to impute SV into the large cohorts of animals genotyped using the existing SNP arrays. 
However, the majority of whole-genome sequence (WGS)-based studies in cattle were either constrained by (small) sample size (Shin et al., 2014; Gao et al., 2017; Mielczarek et al., 2017) or (unavailability of) SV genotypes (Chen et al., 2017). Therefore, building a suitable reference panel for imputing SV into large cohorts of SNP array-typed cattle population remains a challenge. Earlier, we reported $\sim 8,500$ large deletions (size: 199-773 kb) with genotype likelihoods in 175 WGS animals from 3 Nordic dairy cattle breeds, namely, 67 Nordic Holstein (HOL), 27 Danish Jersey (JER), and 81 RDC (Mesbah-Uddin et al., 2017). We were interested to extend our large deletion (deletion, hereafter) reference panel of 175 animals by including additional sequenced animals from corresponding breeds from shared variant call format (VCF) files of the 1,000 Bull Genomes Project (1KBGP). In this study, we present a strategy for computing deletion genotype likelihoods using the SNP read depth (RD) information from a VCF file when access to BAM files are not available, building a haplotype reference panel and jointly imputing WGS SNP, indels, and deletions.

\section{MATERIALS AND METHODS}

\section{SNP Genotype Data Set}

A total of 13,307 JER, HOL, and RDC animals were genotyped using either Illumina BovineSNP50 (50k, hereafter) or BovineHD BeadChip (777k, hereafter; Illumina Inc., San Diego, CA). The 50k and the $777 \mathrm{k}$ data sets comprised 9,932 (838 JER, 5,371 HOL, and 3,723 RDC) and 3,375 (835 JER, 1,215 HOL, and 1,325 RDC) animals, respectively.

From both data sets, we excluded SNP that were monomorphic, had a GenCall $<0.60$, or deviated from Hardy-Weinberg proportions $\left(P\right.$-value $\left.\leq 1 \times 10^{-5}\right)$ in any of the 3 breeds. We also removed SNP, which overlapped a deletion locus from our deletion data set (see WGS Dataset subsection Deletions). After filtering, the 50k and 777k data sets had 47,237 and 697,296 SNP, respectively.

\section{WGS Data Set}

In total, 772 animals were available in our WGS data set (92 JER, $132 \mathrm{RDC}$, and $548 \mathrm{HOL}$ ). Among these animals, 175 and 597 were from the Nordic sequence project (Brøndum et al., 2014) and the Run-6 of the 1KBGP (Daetwyler et al., 2014), respectively. The GATK v1.6 (McKenna et al., 2010) and SAMtools 0.1.18 mpileup (Li et al., 2009) software were used to call SNP and indels from the Nordic and the 1KBGP
WGS data set, respectively [for details, see Brøndum et al. (2014) and Daetwyler et al. (2014)].

SNP and Indels. In this study, when merging the data, we only considered Phred-scaled genotype likelihoods (PL) for biallelic SNP and indels that occurred in both the Nordic and the 1KBGP data set. We removed a marker from the data set when any of the following conditions was met, namely: (1) minor allele count (MAC) less than 5 (vcftools'-mac 5), (2) deviation from Hardy-Weinberg proportion in any one breed $\left(P\right.$-value $\left.\leq 1 \times 10^{-5}\right)$, $(3)$ missing genotypes in more than $10 \%$ of the animals (-max-missing 0.1$)$, (4) marker within $5 \mathrm{bp}$ from one another (-thin 5), (5) marker quality less than 50 (-minQ 50), (6) markers within $100 \mathrm{bp}$ of gaps in the UMD3.1 bovine genome assembly (Zimin et al., 2009), or (7) markers within 1 $\mathrm{kb}$ of a deletion locus from our deletion list (discussed later). After filtering, 14,800,299 biallelic markers remained in our WGS data set: 14,678,220 SNP and 122,079 indels.

Deletions. In addition, Mesbah-Uddin et al. (2017) estimated PL for $\sim 8,500$ deletions on Nordic samples from the sequences aligned to UMD3.1 (BAM files) using GenomeSTRiP-2 software (Handsaker et al., 2011). Among them, we selected 5,798 deletions after a thinning of $100 \mathrm{~kb}$ using VCFtools" "-thin 100000" option (i.e., excluding deletions within $100 \mathrm{~kb}$ distance from one other). From this data set, we extracted deletion PL for the Nordic animals, whereas for the remaining 597 animals, we estimated PL using a modified version of the RD genotyping method used by Mesbah-Uddin et al. (2018), as explained below.

\section{Estimating Deletion Genotype Likelihoods from VCF File}

We estimated genotype likelihoods for the selected deletions on 597 additional animals from the 1KBGP (Run-6) using RD data from VCF file. The average genome-wide sequencing coverage in these additional animals was $\sim 12($ minimum $=2.21$; maximum $=44.52)$.

Calculation of Expected $R D$. Studies showed that $\mathrm{GC}$ bias [i.e., RD variation due to differences in the percentage of guanine $(\mathrm{G})$ and cytosine $(\mathrm{C})$ bases in the region] can confound the inference of copy number (Cn) from RD (Abyzov et al., 2011; Benjamini and Speed, 2012). For the genome assembly UMD3.1, we first calculated the GC\% in bins of $100 \mathrm{bp}$ after excluding potential Cn variable regions, such as sex chromosomes, mitochondrial sequences, unplaced contigs, assembly gaps, repeat sequences, and CNV and SV from the DGVa database (last accessed January 22, 2019, https://www.ebi.ac.uk/dgva). Next, for each animal, 
we extracted RD data from the corresponding genomic intervals from VCF files, and calculated the expected $\mathrm{RD}\left(\mu_{G C}\right)$ and variance $\left(v_{a r}\right)$ for different GC\% (for details, see Supplemental Methods; https://doi.org/ 10.3168/jds.2019-16946). Substantial variations were present in $\mathrm{RD}$ against different $\mathrm{GC} \%$ between samples (Supplemental Figure S1, https://doi.org/10.3168/jds .2019-16946). To account for this heterogeneous GC\% bias for a given deletion locus, the expected RD was assumed to be the average RDs from its GC\% bin, instead of the overall genomic coverage. For example, when interrogating a 10-kb deletion locus with $30 \%$ GC content, we used the genomic average over all 30\% GC bins as the expected RD for each corresponding animal.

Gaussian Mixture Model. To compute genotype likelihoods for a deletion, we first extracted the SNP (say n SNP in total) within the deleted segment and retrieved RD data from the allelic-depth tag of the VCF file. Here, we only considered biallelic SNP with QUAL $\geq 30$ and a thinning of $10 \mathrm{bp}$. Next, we fitted a Gaussian mixture model (GMM) to the data, assuming a linear relationship between the observed RD within the locus and (unobserved) Cn of the locus, such as

$$
p\left(\mathbf{R D}_{i}\right)=\sum_{k=0}^{2} w_{k} N\left(\mathbf{R D}_{i} \mid m u_{k}, v a r_{k}\right) .
$$

Here, $\mathbf{R D}$ is a vector of $\mathrm{n}$ data points corresponding to the n SNP; $p$ is the probability of $i$ th $\mathbf{R D}, k(0$ to 2$)$ is an indicator variable for the Gaussian component; $w_{k}$ is the relative weight for Cn classes (e.g., $w_{0}, w_{1}, w_{2}$ for $C n_{0}=$ homozygous for deletion, $C n_{1}=$ hemizygous, and $C n_{2}=$ homozygous for reference allele, respectively); $m u_{k}$ and $v a r_{k}$ are the expectation and variance of the corresponding Gaussian distribution. The GMM parameters were estimated using an expectation-maximization (EM) algorithm as follows.

Initialization of the EM. Assuming a deletion locus, we constrained GMM to fit exactly 3 components with a fixed $m u_{k}$ for each $k$ such that $m u_{0}=0$ for $C n_{0}$, $m u_{1}=\frac{\mu_{G C}}{2}$ for $C n_{1}$, and $m u_{2}=\mu_{G C}$ for $C n_{2}$. We initialized $w_{k}$ with 3 equal weights of $1 / 3$, and $v_{a r}$ with the value of $0.2, \frac{\operatorname{var}_{G C}}{2}$, and $v a r_{G C}$, for $C n_{0}, C n_{1}$, and $C n_{2}$, respectively.

E-Step. Given the parameters, the expected values for the latent variable $Z_{i k}$ were estimated as

$$
Z_{i k}=\frac{w_{k} N\left(\mathbf{R D}_{i} \mid m u_{k}, \operatorname{var}_{k}\right)}{\sum_{k=0}^{2} w_{k} N\left(\mathbf{R D}_{i} \mid m u_{k}, \operatorname{var}_{k}\right)}
$$

M-Step. The membership weights and variances were updated using the $Z_{i k}$ values from the E-step (while keeping $m u_{k}$ fixed):

$$
\begin{gathered}
w_{k}=\frac{1}{n} \sum_{i=1}^{n} Z_{i k}, \\
\operatorname{var}_{k}=\frac{\sum_{i=1}^{n} Z_{i k}\left(\mathbf{R D}_{i}-m u_{k}\right)^{2}}{\sum_{i=1}^{n} Z_{i k}} .
\end{gathered}
$$

For a given deletion, parameters $w_{k}$ and $v_{a r}$ were estimated iteratively from the observed RD data of each animal using the EM algorithm until convergence. The scripts used to prepare the RD data from the VCF file and to estimate deletion PL could be accessed from https://github.com/MMesbahU/ImputeDelPipeline/ tree/master/read_Depth_genotyping.

Refining the Genotypes. Next, to refine the deletion genotypes along with the WGS SNP and indels, we combined PL estimates for all these variants and performed chromosome-wise phasing using Beagle v4.r1274 software (Browning and Browning, 2016) (hereafter referred to as Beagle; for details see the Phasing section).

Evaluation of the Deletion Genotyping Pipeline. We evaluated the pipeline for Brachyspinaassociated deletion located on chromosome 21 between the positions 21,184,869 and 21,188,202 bp (Charlier et al., 2012). We had 113 Holstein animals with known Brachyspina carrier status: 13 carriers and 100 noncarriers of the deletion. Using the GMM approach described before, we first estimated deletion PL using the SNP RD from the VCF file. After excluding variants within the deletion, we extracted PL for WGS SNP and indels within $\pm 1 \mathrm{Mb}$ of the deletion. Next, to refine the deletion genotypes, we performed phasing using the surrounding haplotypes using Beagle (with same parameters as discussed in the Phasing step). Finally, the genotype concordance (true vs. Beagle's best guess genotypes) was manually inspected.

Next, we evaluated the deletion genotyping pipeline on 144 animals for which both BAM file-based deletion genotype likelihood (Mesbah-Uddin et al., 2017) and the 1KBGP VCF files were available. For these animals, we extracted the best guess deletion genotypes (as true genotype set) from the Beagle-phased reference panel $\left(\operatorname{Ref}_{\text {Beagle }}\right.$; as described in the Refining the Genotypes section). Using the GMM pipeline, we estimated deletion PL using the SNP RD from the 1KBGP VCF file. We combined deletion PL from the GMM step with the PL for WGS SNP and indels within $\pm 1 \mathrm{Mb}$ of the deletion, followed by a genotype-phasing step using Beagle. 
The final evaluation data set comprised a convenience sample of 2,481 deletions with MAC $\geq 1$ in the 144 animals, for which deletion-genotyping were completed after $96 \mathrm{~h}$ of runtime. This data set represents all 29 autosomes with an overall median of 84 deletions, and a minimum and maximum of 48 and 138 deletions from chromosome 1 and 20, respectively. Finally, we performed (Pearson's) correlation and concordance analysis between Beagle's best guess genotypes (converted to allele dosage of $0=$ homozygous reference, $1=$ hemizygous, or 2 = homozygous deletion) from Ref Beagle $_{\text {versus }}$ GMM-based deletion genotyping pipeline.

\section{Phasing}

Following the method used by Delaneau et al. (2014), we phased our genotype data set using a combination of software Beagle and SHAPEIT v2.r837 (Delaneau et al., 2013a; hereafter referred to as SHAPEIT). First, providing the WGS PL as input, we ran Beagle for 10 burn-in and 15 sampling iterations with a window size of 12,000 markers and an overlap of 2,000 markers between consecutive windows. From this step, we obtained posterior genotype probabilities (GP) for each biallelic variant for the 3 possible genotypes [e.g., homozygous reference, heterozygous (or hemizygous for deletion), and homozygous for alternate allele (or homozygous deletion)]. We fixed the genotype for a variant as known when Beagle GP $\geq 0.99$, and for the remaining variants, we re-called the genotype using SHAPEIT. To initialize phasing and calling in SHAPEIT, we used the haplotypes generated in the Beagle step. After this initialization, SHAPEIT was run for 12 pruning stages of 4 iterations each, followed by 20 main Markov chain Monte Carlo sampling iterations. We used a window size of $0.1 \mathrm{Mb}$ for the WGS variants, and $2 \mathrm{Mb}$ for the $50 \mathrm{k}$ and $777 \mathrm{k}$ variants, following the SHAPEIT guidelines (last accessed May 8, 2019, https://mathgen .stats.ox.ac.uk/genetics_software/shapeit/shapeit.html \#gcall). In each window, 600 conditioning haplotypes were generated, among which, 400 were Hamming distance conditioning (-states 400) and the remaining 200 were chosen randomly (-states-random 200) to improve the mixing of the Markov chain Monte Carlo. However, both the $50 \mathrm{k}$ and $777 \mathrm{k}$ data set were already phased using Beagle. We took the Beagle haplotypes and GP, and performed phasing (and calling) using SHAPEIT, as described above.

\section{Imputation}

The imputation was performed in 2 steps using Minimac3 v.2.0.1 software (Das et al., 2016; hereafter referred to as Minimac) with default parameters. In the first step, we imputed 9,932 animals from $50 \mathrm{k}$ to $777 \mathrm{k}$ using the $777 \mathrm{k}$ haplotype reference panel consisting of 3,375 animals. Few animals were common in both the $50 \mathrm{k}$ and $777 \mathrm{k}$ data set or $777 \mathrm{k}$ and WGS data set. Here, we kept the sample with highest genotype density (i.e., WGS $>777 \mathrm{k}>50 \mathrm{k}$ ) in the reference panel and remove the sample from the corresponding imputation target data set. We then combined both imputed and genotyped $777 \mathrm{k}$ data set to prepare a nonredundant target of 12,966 animals (1,640 JER, 6,371 HOL, and $4,955 \mathrm{RDC})$, after excluding the overlap between the 777k and WGS data set. Next, using the haplotype reference panel of 772 WGS animals we imputed our target population from $777 \mathrm{k}$ to WGS SNP, indels, and deletions.

\section{Evaluation of Imputation Accuracy}

First, we prepared a phased reference panel using Beagle with the following inputs: PL for WGS SNP and indels in 772 animals, deletion PL in 175 animals from the study by Mesbah-Uddin et al. (2017), deletion PL estimated for 597 animals using auxiliary RD data from VCF files with missing deletion PL coded as "." for EM nonconvergence. Second, to get the final reference panel, we re-phased and refined genotypes at loci with Beagle GP less than 0.99 using SHAPEIT. We refer to the first and second reference panels as Ref $_{\text {Beagle }}$ and Ref $f_{\text {Beagle_SHAPEIT }}$, respectively. We assessed the performance of this 2-step approach on chromosome 29. The performance differences between the 2 reference panels, $\operatorname{Ref}_{\text {Beagle_SHAPEIT }}$ versus $\operatorname{Ref}_{\text {Beagle, were }}$ tested using 2-tailed paired $t$-test.

Cross Validation. We prepared 2 reference panels

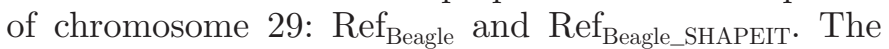
leave-out trials were performed on 112 deletions with $\mathrm{MAC} \geq 5$ common in both data sets. For each deletion, we extracted WGS markers within $1 \mathrm{Mb}$ on either side of the deletion from the $\operatorname{Ref}_{\text {Beagle }}$ or Ref $f_{\text {Beagle_SHAPEIT, }}$ after excluding markers within and $\pm 500 \mathrm{bp}$ surrounding regions. Next, we removed genotype phasing for all the markers selected from the corresponding reference panel (i.e., $R f_{\text {Beagle }}$ or $\operatorname{Ref}_{\text {Beagle_SHAPEIT }}$ ), and performed 77 leave-out trials by randomly masking deletion genotypes for a set of nonoverlapping (i.e., sampled without replacement) 10 animals in each trial (12 animals in the last trial). We used the same random seed to mask the genotypes of samples in both data set. These analyses were performed using Beagle with the same parameters as in the "Phasing" step.

Here, we considered the deletion genotypes in the corresponding reference panel as the true genotype for 
that scenario. To evaluate the imputation accuracy at each deletion locus, we extracted the imputed and true genotypes for the corresponding animals from the 77 trials, and performed Pearson correlation (r) of allele dosages (coded as 0,1 , or 2 for homozygous reference, hemizygous, or homozygous deletion, respectively) between the reference and the imputed data set. Finally, we reported the squared version of the correlation coefficient $\left(\mathrm{r}^{2}\right)$ in the Results section.

Downstream Imputation Performance. For chromosome 29, we also assessed downstream performances of the 2 reference panels $\left(\operatorname{Ref}_{\text {Beagle }}\right.$ and Ref $_{\text {Beagle_SHAPEIT }}$ ) to impute jointly the target $777 \mathrm{k}$ data set to WGS SNP, indels, and deletions using Minimac. Here, we evaluated the performance based on the imputation accuracy estimates $\left(\hat{r}^{2}\right)$ provided in the Minimac "info" output file. Under the assumption that genotype counts for a poorly imputed marker will shrink toward its expectation, Minimac calculates imputation accuracy as follows (last accessed May 8, 2019, https:// genome.sph.umich.edu/wiki/Minimac3_Info_File \#Rsq):

$$
\hat{r}^{2}=\frac{\frac{1}{2 n} \times \sum_{i=1}^{2 n}\left(D_{i}-\hat{p}\right)^{2}}{\hat{p}(1-\hat{p})} .
$$

Here, $\hat{p}=$ the estimated alternate allele frequency, $D_{i}=$ the imputed alternate allele probability at the $i$ th haplotype, and $n=$ the number of samples in the imputation target panel.

Imputation Accuracy at 2 Known Deletion Loci in $R D C$. We evaluated the accuracy of imputation for 2 deletions, esv4015629 (Chr12: 20,100,648-20,763,119 bp) and esv4019342 (Chr23: 12,291,761-12,817,087 bp), that segregate in the RDC population. We imputed genotypes for these 2 deletions (along with other deletions) in this study using the Ref $\mathrm{Beagle}_{\text {BSHAPEIT }}$ reference panel of 772 animals. We also had 50k chip intensitybased deletion genotypes from Kadri et al. (2014) on 4,378 animals for esv4015629 locus and Sahana et al. (2016) on 3,864 animals for esv4019342 locus [for genotyping method, see Supplementary Text S1 in Kadri et al. (2014)]. We performed correlation (allele dosages) and (genotype) concordance analysis between imputed and chip intensity-based deletion genotypes.

\section{Tools Used for Data Manipulation and Analyses}

We used following tools for data preparation and analyses: R software version 3.1.2 (R Core Team, 2014), Python software version 3.7 (https://www.python.org/),
VCFtools v0.1.12a (Danecek et al., 2011), BEDTools v2.26.0 (Quinlan and Hall, 2010), and BCFtools v1.7 (https://github.com/samtools/BCFtools).

\section{Principal Component Analysis}

To distinguish the 3 breeds present in our data set, we performed a principal component (PC) analysis using the imputed WGS variants with minor allele frequency $(\mathbf{M A F})>1 \%$. Within this MAF threshold, we randomly selected $1 \%$ SNP, $10 \%$ indels, and $100 \%$ deletions from each of the 29 autosomes. The final data set contained 137,064 SNP, 11,710 indels, and 4,670 deletions. The analysis was performed using PLINK (v2.00a2 AVX2) software (Chang et al., 2015). For each of the 3 variant classes, we presented the first $2 \mathrm{PC}$ and along with the proportion of variance explained by the corresponding PC.

\section{RESULTS AND DISCUSSION}

\section{Deletion Genotyping Using RD Data from the 1KBGP VCF File}

The WGS reference panel was extended from 175 animals to 772 animals by data from the 1KBGP. In the extended reference panel, genotype likelihoods (PL) for 14,800,299 biallelic markers (SNP and indels) on all the animals were available. However, the deletion PL for 5,798 selected markers were only available on 175 animals. For the remaining 597 animals, we estimated PL by fitting the GMM with 3 components to the RD data extracted from the VCF file. We then used surrounding haplotypes to refine deletion genotypes in animals with ambiguous (or missing) PL estimates.

We evaluated the deletion genotyping pipeline for classifying animals for their carrier status for the brachyspina-associated deletion (Charlier et al., 2012) in a panel of 113 animals with known carrier status including 13 hemizygotes and 100 homozygotes for the reference allele. We found that our 2-step approachestimating PL using WGS SNP RD data from the VCF file followed by a genotype refinement step using Beagle - accurately classified deletion genotypes in all the animals (13 hemizygotes and 100 homozygotes for the reference allele).

We next evaluated the genotyping pipeline on 2,481 deletions in a panel of 144 animals for which both sequence-based (BAM file) as well as GMM-based deletion genotypes were available. We found that using our approach, we could classify deletion genotypes with high accuracy: the median (allele dosage) correlation and (genotype) concordance between the true versus 
GMM-based deletion genotypes was 0.896 and 0.972 , respectively (Table 1 ).

\section{WGS Reference Panel of 772 Animals}

Following previous reports (Brøndum et al., 2014; Pausch et al., 2017), where the authors showed that a multi-breed reference panel provides better downstream imputation performance in cattle compared with a single-breed reference panel, we built a multi-breed WGS reference panel of 772 animals that included 92 JER, 132 RDC, and 548 HOL. We applied the GMM pipeline to estimate the genotype likelihoods for all the deletions in the additional 597 animals included from the 1KBGP. Next, to refine the genotypes and get posterior GP, we performed phasing by chromosome using Beagle with the PL estimates for all SNP, indels, and deletions as inputs.

However, studies in humans have shown that an initial phasing of WGS genotypes using Beagle followed by fixing the genotype with high posterior probability (e.g., Beagle GP > 0.995) and re-calling the remaining markers genotypes using SHAPEIT "-call" provides further improvement in the reference panel (Delaneau et al., 2014; 1000 Genomes Project Consortium et al., 2015). Besides, SHAPEIT can also improve haplotype phasing by leveraging on the haplotype sharing within and between populations with heterogeneous ancestry (Delaneau et al., 2013b). Here, both our WGS reference and SNP array-typed target populations consist of animals with diverse ancestry [see Figure 3 from Bouwman et al. (2018) for examples of haplotype diversity in JER, HOL, and RDC; see Zhang et al. (2018) for a detailed account in RDC]. In addition, on average $\sim 2.7 \%$ of the total WGS genotypes in our data set had Beagle GP <0.99 (Supplemental Table S1, https://doi

Table 1. Validation of the Gaussian mixture model (GMM)-based deletion genotyping pipeline (2,481 deletions and 144 animals)

\begin{tabular}{lcc}
\hline \multirow{2}{*}{$\begin{array}{l}\text { Summary } \\
\text { statistics }\end{array}$} & True $^{1}$ vs. GMM-based deletion genotypes \\
\cline { 2 - 3 } & Pearson correlation & Concordance \\
\hline Median & 0.896 & 0.972 \\
Mean & 0.768 & 0.910 \\
1st quartile & 0.636 & 0.910 \\
3rd quartile & 0.981 & 0.993 \\
Minimum & -0.355 & 0.035 \\
Maximum & 1.000 & 1.000 \\
\hline
\end{tabular}

${ }^{1}$ True genotype data set comprised deletion Phred-scaled genotype likelihoods (PL) estimated from whole-genome sequence BAM files using GenomeSTRiP followed by chromosome-wise genotype refinement using Beagle v4.r1274 software (Browning and Browning, 2016).

${ }^{2}$ Estimation of deletion PL using GMM, followed by genotype refinement using surrounding SNP haplotypes $( \pm 1 \mathrm{Mb})$ using Beagle. .org/10.3168/jds.2019-16946). Furthermore, there were $7.3 \%$ (1.1 of 14.7 million) SNP, $18.3 \%$ (22.4 of $122.1 \mathrm{k}$ ) indels, and $66.6 \%$ (3.9 of $5.8 \mathrm{k}$ ) deletions where more than 10\% genotypes were below this threshold (Supplemental Table S1, https://doi.org/10.3168/jds.2019 -16946). Therefore, we expected that by using SHAPEIT we could further improve the reference panel by improving (low-quality) genotype calls and haplotype phasing. Based on this assumption, we next refined the reference panel using SHAPEIT, where all genotypes were re-phased, and re-called when Beagle GP was less than 0.99 .

To evaluate the effect of this 2-step refinement approach on imputation accuracy we prepared $\operatorname{Ref}_{\text {Beagle }}$ and Ref $f_{\text {Beagle_SHAPEIT }}$ of the chromosome 29. First, we performed leave-out trials on 112 deletions with MAC $\geq 5$ from this chromosome and evaluated the imputation accuracy using (squared) Pearson correlation $\left(\mathrm{r}^{2}\right)$ between true versus imputed dosages of 0 , 1 , or 2 for homozygous reference, hemizygous, or homozygous deletion, respectively (see Cross Validation section in Materials and Methods). After the trials, 5 deletions became homozygous for the reference allele. These were excluded from subsequent analysis. Overall, the average gain in accuracy was $\mathrm{r}^{2}=0.081$ (95\% CI: $\left.0.053,0.109\right)$ with $\operatorname{Ref}_{\text {Beagle_SHAPEIT }}$ versus $\operatorname{Ref}_{\text {Beagle }}\left(P=7.9 \times 10^{-8}\right.$; 2 -tailed paired $t$-test). The performance gain was even higher $\left[\mathrm{r}^{2}=0.105\right.$ (CI: $\left.\left.0.069,0.141\right), P=1.7 \times 10^{-7}\right]$ for variants where more than $10 \%$ of the genotypes were under the GP threshold (Table 2). Furthermore, 76 versus $90 \%$ of the deletions achieved $\mathrm{r}^{2}>0.80$ using the reference $\operatorname{Ref}_{\text {Beagle }}$ vs. Ref $f_{\text {Beagle_SHAPEIT }}$, respectively (Figure 1a).

Next, we evaluated the downstream performances of the 2 reference panels to impute jointly a target population of $\sim 13 \mathrm{k}$ animals with $777 \mathrm{k}$ genotypes to WGS SNP, indels, and deletions of chromosome 29 using Minimac. As expected, the reference panel $\operatorname{Ref}_{\text {Beagle_SHAPEIT }}$ showed better downstream imputation performance for all variant types compared with that of $\operatorname{Ref}_{\text {Beagle }}$ (Figures $1 \mathrm{~b}$ and 2ab, and Table 2). The average gains in accuracy in terms of Minimac $\mathrm{r}^{2}$ were 0.032 (CI: $0.012,0.053 ; P$ $=0.002$ ) for deletions, 0.020 (CI: 0.019, 0.020; $P<1.0$ $\times 10^{-16}$ ) for SNP, and 0.034 (CI: 0.031, 0.037; $P<1.0$ $\times 10^{-16}$ ) for indels (Table 2). However, after Beagle step, $\sim 8 \%$ of the SNP, $20 \%$ of the indels, and $70 \%$ of the deletions had more than $10 \%$ of genotypes below the GP threshold (Supplemental Table S1, https://doi .org/10.3168/jds.2019-16946). Indeed, for these markers we observed a clear difference between before versus after the SHAPEIT step. For example, the average gain in accuracy after the SHAPEIT step was 0.041 (CI: 0.011, 0.070; $P=0.007$ ) for deletions, 0.058 (CI: 0.056, 
$0.059 ; P<1.0 \times 10^{-16}$ ) for SNP, and 0.076 (CI: 0.065, $0.087 ; P<1.0 \times 10^{-16}$ ) for indels (Table 2). Besides, 87 versus $93 \%$ of the deletions (Figure $1 \mathrm{~b}$ ), 87 versus $89 \%$ of the SNP (Figure 2a), and 78 versus $83 \%$ of the indels (Figure $2 \mathrm{~b}$ ) achieved $\mathrm{r}^{2}>0.80$ using the reference panel Ref $f_{\text {Beagle }}$ versus $\operatorname{Ref}_{\text {Beagle_SHAPEIT, }}$, respectively. It is worth noting that for the majority of the imputed variants, allele frequencies differed between the 2 reference panels. Nevertheless, the average $r^{2}$ in different $5 \%$ MAF bins were also higher with $\operatorname{Ref}_{\text {Beagle_SHAPEIT }}$ versus $\operatorname{Ref}_{\text {Beagle }}$ (Supplemental Figure S2 and Table S2, https://doi.org/10.3168/jds.2019-16946). Thus, it is likely that SHAPEIT "-call" step improved genotype calls, providing an improved WGS reference panel for the chromosome. These results are in agreement with the reported performance gains in humans (Delaneau et al., 2014).

Finally, we evaluated the accuracy of imputed genotypes for esv4015629 (Chr12:20,100,648-20,763,119 bp) and esv4019342 (Chr23: 12,291,761-12,817,087 bp) deletions that segregate in the RDC population with a (imputed) MAF of 0.137 and 0.052 , respectively. The Pearson correlation between imputed versus 50k chip intensity-based allele dosage was 0.913 for esv4015629 and 0.956 for esv4019342. The genotype concordance between the 2 data sets was 0.965 for esv4015629 and 0.991 for esv4019342. It is noteworthy that in the imputed data set, for esv4019342, we only observed hemizygotes, whereas for esv4015629, we found 9 carriers that were imputed as homozygotes for the deletion. For esv4015629, we next analyzed the surrounding SNP haplotypes (build using imputed 777k data) and found the homozygous haplotype in both sides of the deletion. This indicates both ancestral haplotype (without deletion) and the haplotype with deletion segregating in the RDC population, which was previously reported by Kadri et al. (2014; for detail, see Supplementary Figure S2 in Kadri et al., 2014).

\section{Imputation of WGS SNP, Indels, and Deletions to the Chip-Typed Cattle Population}

Our imputation target was a population of $\sim 13 \mathrm{k}$ animals (1,640 JER, 6,371 HOL, and 4,955 RDC) that were genotyped using either the $50 \mathrm{k}$ or the $777 \mathrm{k}$ BeadChip. We prepared this data set using the same pipeline: 2-step phasing followed by imputation from the $50 \mathrm{k}$ to the $777 \mathrm{k}$ genotypes using Minimac (see Phasing and Imputation sections in Materials and Methods). Next, for the 29 bovine autosomes, we jointly imputed this target $777 \mathrm{k}$ data set to WGS SNP, indels, and deletions using the reference panel Ref $f_{\text {Beagle_SHAPEIT }}$ comprising 772 animals. In Figure $4 \mathrm{a}-\mathrm{c}$, we present the MAF versus Minimac $r^{2}$ estimates for the imputed 
(a) Leave-out trials

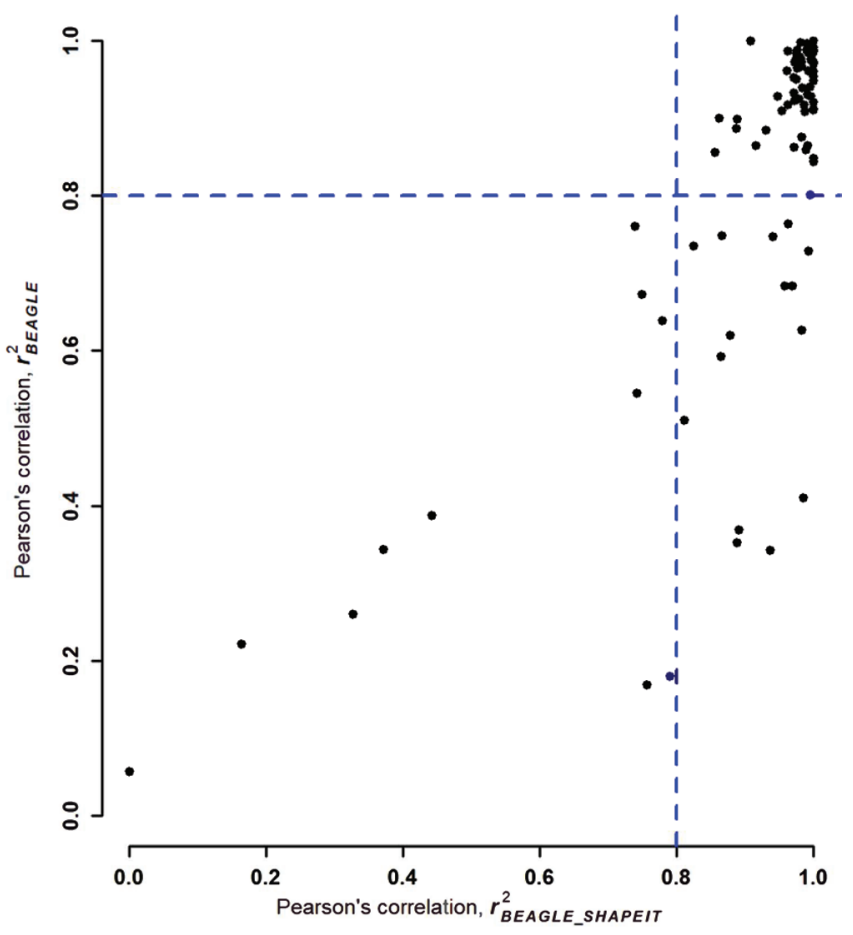

(b) Downstream performance

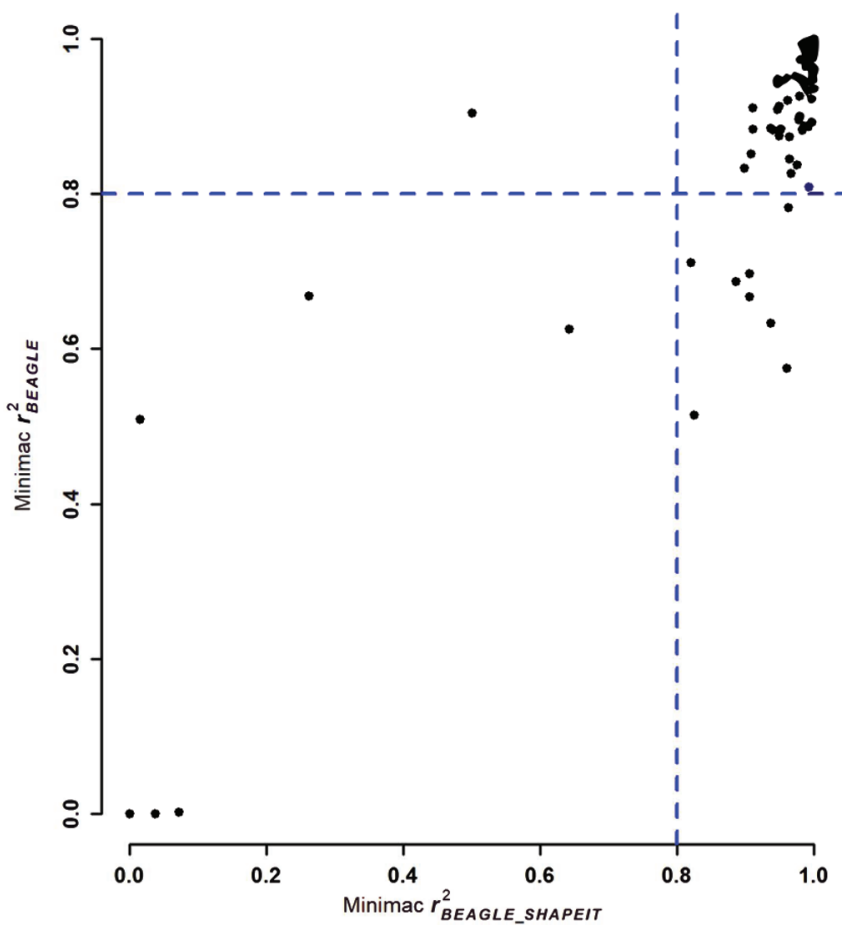

Figure 1. Imputation accuracy for 107 deletions on chromosome 29 using the reference panels Ref $f_{\text {Beagle }}$ versus Ref Beagle_SHAPEIT. $_{\text {(a) Leave-out }}$ trials. For each deletion, 77 trials were performed: 76 trials of 10 animals each and 1 trial of 12 animals. Imputation accuracy was calculated using the squared Pearson correlation $\left(\mathrm{r}^{2}\right)$ between the true and imputed alternative allele dosages. (b) Downstream performance. Using the 2 reference panels, a target population of $\sim 13 \mathrm{k}$ animals with the $777 \mathrm{k}$ genotypes was imputed for the deletions (along with other whole-genome sequence markers) of chromosome 29 using the Minimac3 v.2.0.1 software (Das et al., 2016). Each dot represent one deletion; dashed lines indicate an $\mathrm{r}^{2}$ value of 0.80 . Ref $\mathrm{Beagle}_{\text {e }}=$ reference panel phased using Beagle v4.r1274 software (Browning and Browning, 2016); Ref Beagle_SHAPEIT $_{2}=$ reference panel phased using Beagle followed by re-phasing using SHAPEIT v2.r837 (Delaneau et al., 2013a). 

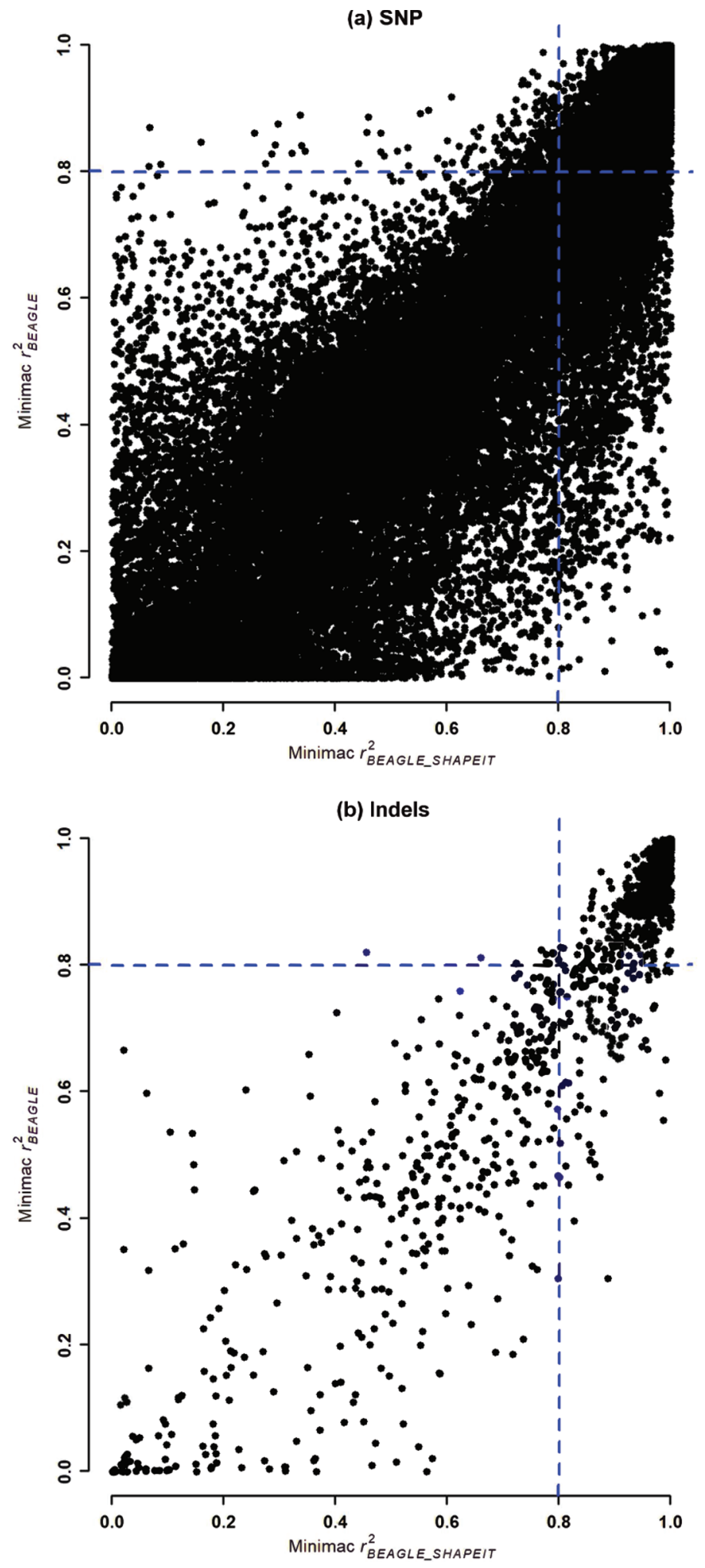

Figure 2. Downstream imputation performance between $\operatorname{Ref}_{\text {Beagle }}$ versus Ref $_{\text {Beagle_SHAPEIT }}$ on test chromosome 29. (a) SNP. In total, 326,838 SNP were imputed from this chromosome; each dot represents a SNP. (b) Insertions and deletions (indels). There were 2,723 imputed indels in this chromosome; each dot represents an indel. The x- and y-axes represent corresponding Minimac3 v.2.0.1 software (Das et al., 2016) squared Pearson correlation $\left(\mathrm{r}^{2}\right)$ value; dashed lines indicate Minimac $\mathrm{r}^{2}$ value of 0.80 . Ref $\mathrm{Beagle}_{\text {e }}=$ reference panel phased using Beagle v4.r1274 soft-

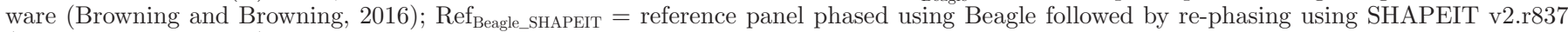
(Delaneau et al., 2013a). 
WGS markers. The average imputation accuracy for SNP, indels, and deletions were 0.928 (SD 0.207), 0.900 (0.217), and 0.904 (0.220), respectively. Here, irrespective of the marker type, the common variants (MAF $>5 \%)$ were well imputed with an average $\mathrm{r}^{2}>0.90$ (Table 3). Furthermore, low-frequency variants $(1 \% \leq$ MAF $\leq 5 \%)$ were also well imputed with an average $\mathrm{r}^{2}$ value of 0.889 (0.250) for SNP, 0.874 (0.245) for indels, and $0.927(0.154)$ for deletions. As for the rare variants (MAF $<1 \%$ ), we observed a sharp reduction in imputation accuracy compared with that of low frequency variants: differences of $0.361,0.325$, and 0.214 were seen for SNP, indels, and deletions, respectively (Table 3). Similar low imputation accuracies for rare variants were also reported previously (Brøndum et al., 2014; Pausch et al., 2017). Rare variants are often novel in the population, have low linkage-disequilibrium with neighboring markers, and are poorly represented in the reference panel, if at all. For these variants, we further stratified the $\mathrm{r}^{2}$ into MAF bins of $0.1 \%$ (Table $3)$. We found that both the SNP and indels with MAF $>0.5 \%$ had an imputation accuracy $\mathrm{r}^{2}>0.50$. The reduction was mainly due to variants in the bottom half (in terms of MAF) of this category. In contrast to SNP and indels, rare deletions with $\mathrm{MAF}=0.2 \%$ were also imputed with high accuracy $\left(\mathrm{r}^{2}=0.633\right)$, and for the lowest $0.1 \% \mathrm{MAF}$ bin (i.e., $0<\mathrm{MAF} \leq 0.1 \%$ ), $\mathrm{r}^{2}$ was 0.313 (Table 3). Nevertheless, compared with the previous report on this population [see Figure S2 from $\mathrm{Wu}$ et al. (2016)], we observed substantial gains in accuracy in a wide range of MAF bins (Figure 3, Table 3 and Supplemental Table S3, https://doi.org/10 $.3168 /$ jds.2019-16946). These results also showed that, given a suitable reference panel, Minimac could jointly impute various classes of biallelic sequence variants - in a frequency spectrum range from rare to common- to the existing chip-typed population with high accuracy.

$P C$ Analysis. Next, to assess the usability of this data set in population-genetic studies, we performed PC analysis using a random sample of the imputed variants with MAF $>1 \%$. Here we verified that the 3 classes of imputed markers were able to distinguish the 3 populations present in our data set in a similar way. Indeed, with the top 2 PC from SNP, indels, and deletions, we were able to illustrate the population structure present in our data set (Figure 4).

Limitations. We acknowledge that our current approach is not suitable for imputation of all classes of SV. For example, using the GMM-based genotyping pipeline outlined in this study, one could only genotype a locus with Cn loss (e.g., deletion of one or both copies) or gain (not shown in this study), since this approach interrogates SNP RD variation from the VCF file. Furthermore, the success of this GMM approach
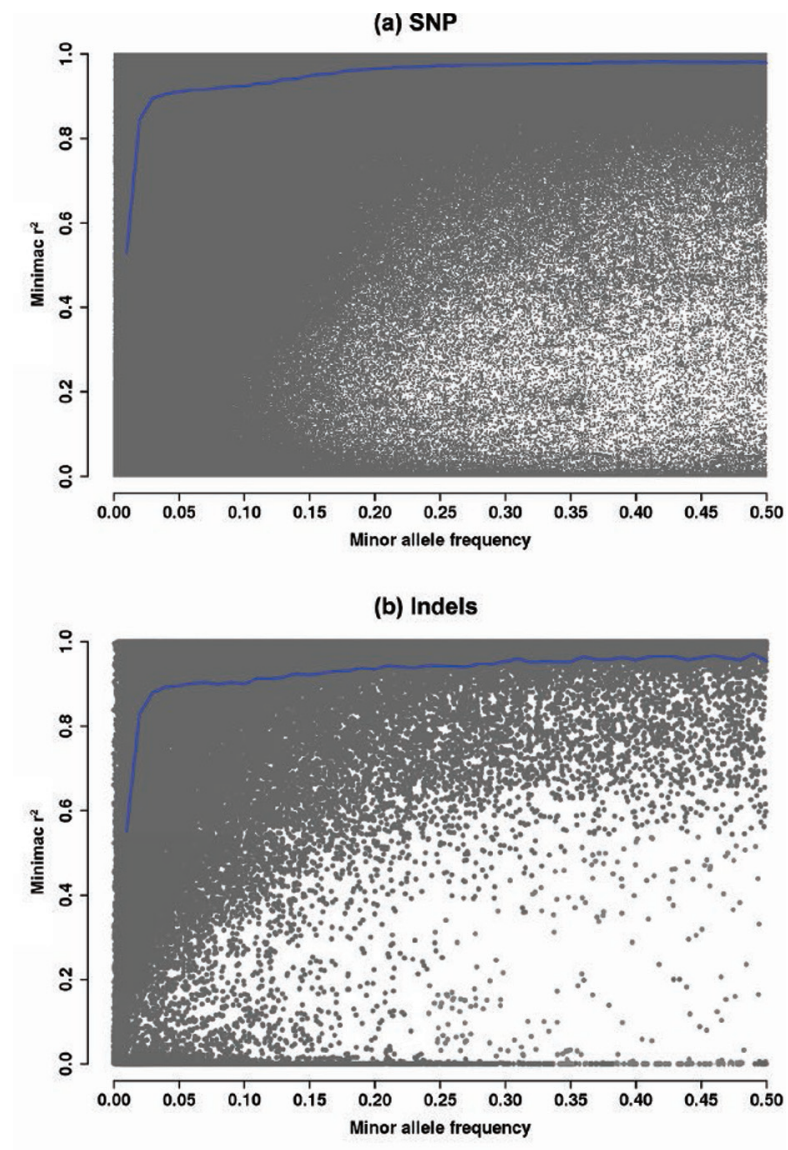

(c) Deletions

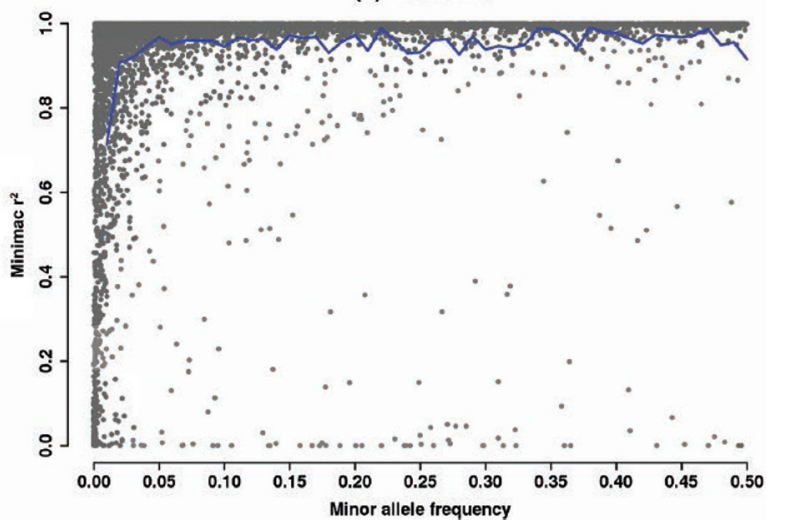

Figure 3. Imputation accuracy of whole-genome sequence (WGS) SNP, insertions and deletions (indels), and deletions on the 29 bovine autosomes. Each dot represents an imputed marker with minor allele frequency $(\mathrm{MAF})>0$. There are (a) 14,070,960 SNP, (b) 122,054 indels, and (c) 5,730 deletions. Here, the imputation accuracy was greater than 0.80 for $91 \%$ SNP, $85 \%$ indels, and $87 \%$ deletions. The solid line in each scatterplot represents the average Minimac3 v.2.0.1 software (Das et al., 2016) squared Pearson correlation $\left(\mathrm{r}^{2}\right)$ in MAF bins of $1 \%$ (see Supplemental Table S3, https://doi.org/10.3168/jds $.2019-16946$, for corresponding $\mathrm{r}^{2}$ values with SD). For the imputed variants, the Pearson correlation coefficients ( $r$ ) between the MAF in the reference panel versus the target population were 0.98 for SNP, 0.97 for indels, and 0.98 for deletions. The observed average differences in MAF, between target versus reference, were 0.0023 (2-tailed $t$-test $P$ $\left.<1.0 \times 10^{-16}\right)$ for SNP, $0.0036\left(P=6.7 \times 10^{-12}\right)$ for indels, and 0.0009 $(P=0.73)$ for deletions. 

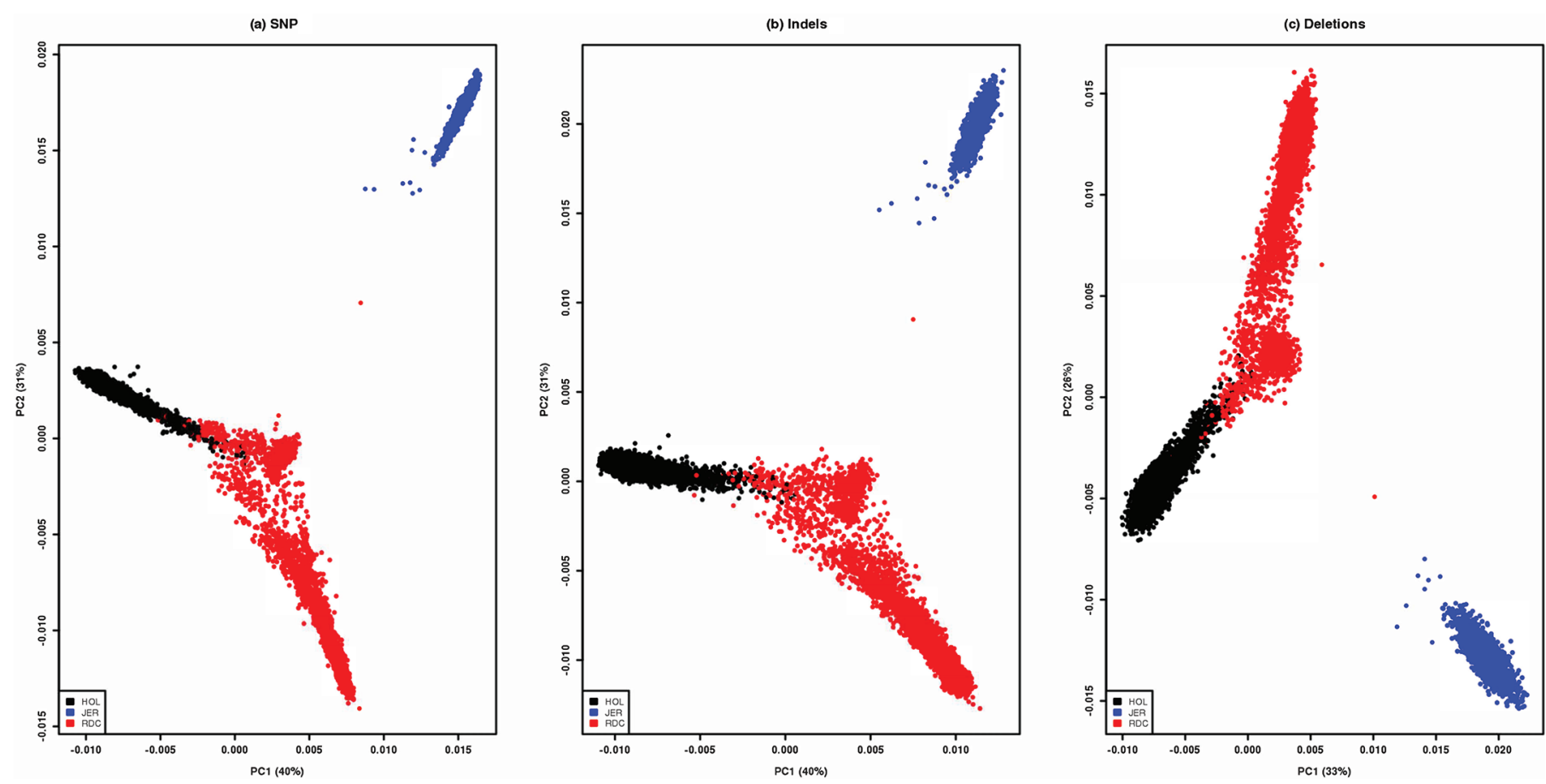

Figure 4. Principal component analysis using imputed whole-genome sequence (WGS) SNP, insertions and deletions (indels), and deletion. Here, the analyses were performed on (a) $\sim 137 \mathrm{k}$ SNP, and (b) $\sim 12 \mathrm{k}$ indels, and (c) $\sim 4.5 \mathrm{k}$ deletions using PLINK (v2.00a2 AVX2) software (Chang et al., 2015). These variants were randomly selected from the imputed WGS variants with minor allele frequency $>1 \%$. PC $=$ principal component; HOL $=$ Holstein; JER $=$ Jersey; RDC $=$ Nordic Red Dairy cattle.

will heavily depend on the sequencing coverage, size of CNV region, and more importantly availability of SNP within the interrogated region. However, it is important to note that BAM files are not always available for the shared genomic resources and there is a need for methods adapted to VCF files. We also acknowledge that our study lacks a comprehensive analysis regarding different aspects of genotype phasing and imputation. Here, we neither explored exhaustively all possible combinations of tools, nor explored the effect of withinbreed versus multi-breed reference panel, on imputation accuracy. We recognize that a within-breed reference

Table 3. Accuracy of imputation from the 777k genotypes to the whole-genome sequence (WGS) SNP, insertions and deletions (indels), and deletions of the 29 bovine autosomes ${ }^{1}$

\begin{tabular}{lccc}
\hline & \multicolumn{3}{c}{ Minimac r $^{2}(\mathrm{SD})$} \\
\cline { 2 - 4 } Category & SNP & Indels & Deletions \\
\hline Overall & $0.928(0.207)$ & $0.900(0.217)$ & $0.904(0.220)$ \\
Common variants (MAF $>5 \%)$ & $0.954(0.163)$ & $0.927(0.176)$ & $0.957(0.148)$ \\
Low frequency variants $1 \leq$ MAF $\leq 5 \%)$ & $0.889(0.250)$ & $0.874(0.245)$ & $0.927(0.154)$ \\
Rare variants (MAF < 1\%) & $0.527(0.392)$ & $0.549(0.370)$ & $0.713(0.343)$ \\
$0.1 \%$ & $0.119(0.183)$ & $0.173(0.222)$ & $0.313(0.365)$ \\
$0.2 \%$ & $0.201(0.231)$ & $0.262(0.265)$ & $0.633(0.364)$ \\
$0.3 \%$ & $0.275(0.269)$ & $0.349(0.271)$ & $0.785(0.280)$ \\
$0.4 \%$ & $0.330(0.306)$ & $0.388(0.294)$ & $0.764(0.294)$ \\
$0.5 \%$ & $0.443(0.348)$ & $0.498(0.328)$ & $0.822(0.243)$ \\
$0.6 \%$ & $0.503(0.371)$ & $0.552(0.351)$ & $0.819(0.217)$ \\
$0.7 \%$ & $0.601(0.370)$ & $0.617(0.353)$ & $0.846(0.232)$ \\
$0.8 \%$ & $0.653(0.377)$ & $0.671(0.356)$ & $0.803(0.275)$ \\
$0.9 \%$ & $0.718(0.355)$ & $0.691(0.350)$ & $0.880(0.180)$ \\
$1.0 \%$ & $0.731(0.357)$ & $0.722(0.341)$ & $0.871(0.207)$ \\
\hline
\end{tabular}

${ }^{1}$ Total number of imputed WGS SNP $=14,070,960$, indels $=122,054$, and deletions $=5,730$. Minimac $=$ Minimac3 v.2.0.1 software (Das et al., 2016); $\mathrm{r}^{2}=$ squared Pearson correlation; MAF $=$ minor allele frequency. 
panel could provide better imputation performance for breed-specific rare variants provided adequate withinbreed reference data; however, we did not perform any focused study for such variants.

\section{CONCLUSIONS}

In this study, we presented strategies to incorporate large chromosomal deletions in population genetic studies, along with SNP and indels. First, we showed an approach to estimate deletion genotype likelihood using the RD data from the VCF file. Second, using a 2-step genotype refinement, we built WGS reference panels that included SNP, indels, and deletions. Finally, we imputed our $777 \mathrm{k}$ population to full sequence. We found that taking genotype uncertainty in the reference panel into account leads to substantial gains in accuracy. These results highlighted the feasibility of building a WGS reference panel comprising different classes of sequence variants and jointly imputing them to the existing SNP chip-typed large cohorts of animals. In addition, we showed that, as WGS resources, such as the 1KBGP, both include animals with shallow and deep sequencing depth, refining the low-confidence genotypes yielded a substantial gain in imputation accuracy. Furthermore, such an approach also provided accurate genotypes for rare variants, which often include putative causal variants. This, in turn, is expected to improve downstream association signals. This study was focused only on the imputations of deletions along with SNP and indels. With such an accurate imputation procedure even for rare and low frequency variants, it becomes possible to explore their potential biological effects by genomewide association study and to study their predictive ability in genomic evaluation. This requires further investigation and will be presented in another study. It is likely that the strategies and pipelines demonstrated here will facilitate joint imputation of large deletions, SNP, and indels, and subsequent population genetic studies in cattle. Nevertheless, this imputation resource will be used in association mapping and estimation of (genomic) breeding values in Nordic dairy cattle breeds.

\section{ACKNOWLEDGMENTS}

This research was supported by the Center for Genomic Selection in Animals and Plants (GenSAP) funded by Innovation Fund Denmark (grant 0603-00519B). Md Mesbah-Uddin benefited from a joint grant from the European Commission within the framework of the Erasmus-Mundus joint doctorate EGS-ABG. Goutam Sahana acknowledges the GUDP project, LiveCalf (No. 34009-16-1101), from the Ministry of Environment and Food of Denmark. The authors also acknowledge the 1,000 Bull Genomes Project for sharing the VCF files. We are also grateful to the Genotoul bioinformatics platform (http://bioinfo.genotoul.fr/), Toulouse Midi-Pyrenees, France, for providing computing and storage resources. All relevant data are included in the manuscript and its additional files. The WGS SNP and indels are available at http://www.1000bullgenomes .com/doco/1000bulls_v6_annotated_snps.tab.gz, http://www.1000bullgenomes.com/doco/1000bulls _v6_annotated_indels.tab.gz, and large deletions are at ftp://ftp.ebi.ac.uk/pub/databases/dgva/estd234 _Mesbah-Uddin_et_al_2017/. The imputation pipelines are available at https://github.com/MMesbahU/ ImputeDelPipeline.git. MMU, BG, MSL, and GS conceived and designed the study. MMU performed computational analyses. MMU drafted the manuscript. BG, MSL, DB, and GS jointly supervised the study. $\mathrm{BG}, \mathrm{DB}$, and GS critically revised the manuscript. All authors read and approved the final manuscript.

\section{REFERENCES}

Abyzov, A., A. E. Urban, M. Snyder, and M. Gerstein. 2011. CNVnator: An approach to discover, genotype, and characterize typical and atypical CNVs from family and population genome sequencing. Genome Res. 21:974-984. https://doi.org/10.1101/gr.114876 .110 .

Alkan, C., B. P. Coe, and E. E. Eichler. 2011. Genome structural variation discovery and genotyping. Nat. Rev. Genet. 12:363-376. https://doi.org/10.1038/nrg2958.

Benjamini, Y., and T. P. Speed. 2012. Summarizing and correcting the GC content bias in high-throughput sequencing. Nucleic Acids Res. 40:e72. https://doi.org/10.1093/nar/gks001.

Bickhart, D. M., and G. E. Liu. 2014. The challenges and importance of structural variation detection in livestock. Front. Genet. 5:37. https://doi.org/10.3389/fgene.2014.00037.

Boichard, D., M. Boussaha, A. Capitan, D. Rocha, C. Hozé, M. P. Sanchez, T. Tribout, R. Letaief, P. Croiseau, C. Grohs, W. Li, C. Harland, C. Charlier, M. S. Lund, G. Sahana, M. Georges, S. Barbier, W. Coppieters, S. Fritz, and B. Guldbrandtsen. 2018. Experience from large scale use of the EuroGenomics custom SNP chip in cattle. Page 675 in Proc. World Congr. Genet. Appl. Livest. Prod., Auckland, New Zealand. AL Rae Centre for Genetics and Breeding, Massey University, Palmerston North, New Zealand. Accessed May 8, 2019. http://www.wcgalp.org/system/files/ proceedings/2018/experience-large-scale-use-eurogenomics-custom -snp-chip-cattle.pdf.

Boussaha, M., D. Esquerre, J. Barbieri, A. Djari, A. Pinton, R. Letaief, G. Salin, F. Escudie, A. Roulet, S. Fritz, F. Samson, C. Grohs, M. Bernard, C. Klopp, D. Boichard, and D. Rocha. 2015. Genomewide study of structural variants in bovine Holstein, Montbeliarde and Normande dairy breeds. PLoS One 10:e0135931. https://doi .org/10.1371/journal.pone.0135931.

Bouwman, A. C., H. D. Daetwyler, A. J. Chamberlain, C. H. Ponce, M. Sargolzaei, F. S. Schenkel, G. Sahana, A. Govignon-Gion, S. Boitard, M. Dolezal, H. Pausch, R. F. Brøndum, P. J. Bowman, B. Thomsen, B. Guldbrandtsen, M. S. Lund, B. Servin, D. J. Garrick, J. Reecy, J. Vilkki, A. Bagnato, M. Wang, J. L. Hoff, R. D. Schnabel, J. F. Taylor, A. A. E. Vinkhuyzen, F. Panitz, C. Bendixen, L. E. Holm, B. Gredler, C. Hozé, M. Boussaha, M. P. Sanchez, D. Rocha, A. Capitan, T. Tribout, A. Barbat, P. Croiseau, C. Drögemüller, V. Jagannathan, C. Vander Jagt, J. J. Crowley, A. 
Bieber, D. C. Purfield, D. P. Berry, R. Emmerling, K. U. Götz, M. Frischknecht, I. Russ, J. Sölkner, C. P. Van Tassell, R. Fries, P. Stothard, R. F. Veerkamp, D. Boichard, M. E. Goddard, and B. J. Hayes. 2018. Meta-analysis of genome-wide association studies for cattle stature identifies common genes that regulate body size in mammals. Nat. Genet. 50:362-367. https://doi.org/10.1038/ s41588-018-0056-5.

Brøndum, R. F., B. Guldbrandtsen, G. Sahana, M. S. Lund, and G. Su. 2014. Strategies for imputation to whole genome sequence using a single or multi-breed reference population in cattle. BMC Genomics 15:728. https://doi.org/10.1186/1471-2164-15-728.

Browning, B. L., and S. R. Browning. 2016. Genotype imputation with millions of reference samples. Am. J. Hum. Genet. 98:116-126. https://doi.org/10.1016/j.ajhg.2015.11.020.

Chang, C. C., C. C. Chow, L. C. Tellier, S. Vattikuti, S. M. Purcell, and J. J. Lee. 2015. Second-generation PLINK: Rising to the challenge of larger and richer datasets. Gigascience 4:7. https://doi .org/10.1186/s13742-015-0047-8.

Charlier, C., J. S. Agerholm, W. Coppieters, P. Karlskov-Mortensen, W. Li, G. de Jong, C. Fasquelle, L. Karim, S. Cirera, N. Cambisano, N. Ahariz, E. Mullaart, M. Georges, and M. Fredholm. 2012. A deletion in the bovine FANCI gene compromises fertility by causing fetal death and brachyspina. PLoS One 7:e43085. https:// doi.org/10.1371/journal.pone.0043085.

Chen, L., A. J. Chamberlain, C. M. Reich, H. D. Daetwyler, and B. J. Hayes. 2017. Detection and validation of structural variations in bovine whole-genome sequence data. Genet. Sel. Evol. 49:13. https://doi.org/10.1186/s12711-017-0286-5.

Conrad, D. F., D. Pinto, R. Redon, L. Feuk, O. Gokcumen, Y. Zhang, J. Aerts, T. D. Andrews, C. Barnes, P. Campbell, T. Fitzgerald, M. Hu, C. H. Ihm, K. Kristiansson, D. G. Macarthur, J. R. Macdonald, I. Onyiah, A. W. Pang, S. Robson, K. Stirrups, A. Valsesia, K. Walter, J. Wei, C. Wellcome Trust Case Control, C. TylerSmith, N. P. Carter, C. Lee, S. W. Scherer, and M. E. Hurles. 2010. Origins and functional impact of copy number variation in the human genome. Nature 464:704-712. https://doi.org/10.1038/ nature 08516

Daetwyler, H. D., A. Capitan, H. Pausch, P. Stothard, R. van Binsbergen, R. F. Brøndum, X. Liao, A. Djari, S. C. Rodriguez, C. Grohs, D. Esquerre, O. Bouchez, M. N. Rossignol, C. Klopp, D. Rocha, S. Fritz, A. Eggen, P. J. Bowman, D. Coote, A. J. Chamberlain, C. Anderson, C. P. VanTassell, I. Hulsegge, M. E. Goddard, B. Guldbrandtsen, M. S. Lund, R. F. Veerkamp, D. A. Boichard, R. Fries, and B. J. Hayes. 2014. Whole-genome sequencing of 234 bulls facilitates mapping of monogenic and complex traits in cattle. Nat. Genet. 46:858-865. https://doi.org/10.1038/ng.3034.

Danecek, P., A. Auton, G. Abecasis, C. A. Albers, E. Banks, M. A. DePristo, R. E. Handsaker, G. Lunter, G. T. Marth, S. T. Sherry, G. McVean, R. Durbin, and 1000 Genomes Project Analysis Group. 2011. The variant call format and VCFtools. Bioinformatics 27:2156-2158. https://doi.org/10.1093/bioinformatics/btr330.

Das, S., L. Forer, S. Schönherr, C. Sidore, A. E. Locke, A. Kwong, S. I. Vrieze, E. Y. Chew, S. Levy, M. McGue, D. Schlessinger, D. Stambolian, P. R. Loh, W. G. Iacono, A. Swaroop, L. J. Scott, F. Cucca, F. Kronenberg, M. Boehnke, G. R. Abecasis, and C. Fuchsberger. 2016. Next-generation genotype imputation service and methods. Nat. Genet. 48:1284-1287. https://doi.org/10.1038/ ng. 3656 .

Delaneau, O., B. Howie, A. J. Cox, J. F. Zagury, and J. Marchini. 2013a. Haplotype estimation using sequencing reads. Am. J. Hum. Genet. 93:687-696. https://doi.org/10.1016/j.ajhg.2013.09.002.

Delaneau, O., J. Marchini, 1000 Genomes Project Consortium, and 1000 Genomes Project Consortium. 2014. Integrating sequence and array data to create an improved 1000 Genomes Project haplotype reference panel. Nat. Commun. 5:3934. https://doi.org/10 $.1038 /$ ncomms 4934 .

Delaneau, O., J. F. Zagury, and J. Marchini. 2013b. Improved wholechromosome phasing for disease and population genetic studies. Nat. Methods 10:5-6. https://doi.org/10.1038/nmeth.2307.
Gao, Y., J. Jiang, S. Yang, Y. Hou, G. E. Liu, S. Zhang, Q. Zhang, and D. Sun. 2017. CNV discovery for milk composition traits in dairy cattle using whole genome resequencing. BMC Genomics 18:265. https://doi.org/10.1186/s12864-017-3636-3.

Handsaker, R. E., J. M. Korn, J. Nemesh, and S. A. McCarroll. 2011. Discovery and genotyping of genome structural polymorphism by sequencing on a population scale. Nat. Genet. 43:269-276. https:/ /doi.org/10.1038/ng.768.

Handsaker, R. E., V. Van Doren, J. R. Berman, G. Genovese, S. Kashin, L. M. Boettger, and S. A. McCarroll. 2015. Large multiallelic copy number variations in humans. Nat. Genet. 47:296-303. https: //doi.org/10.1038/ng.3200.

Kadri, N. K., G. Sahana, C. Charlier, T. Iso-Touru, B. Guldbrandtsen, L. Karim, U. S. Nielsen, F. Panitz, G. P. Aamand, N. Schulman, M. Georges, J. Vilkki, M. S. Lund, and T. Druet. 2014. A 660-Kb deletion with antagonistic effects on fertility and milk production segregates at high frequency in Nordic Red cattle: additional evidence for the common occurrence of balancing selection in livestock. PLoS Genet. 10:e1004049. https://doi.org/10.1371/journal .pgen.1004049.

Letaief, R., E. Rebours, C. Grohs, C. Meersseman, S. Fritz, L. Trouilh, D. Esquerre, J. Barbieri, C. Klopp, R. Philippe, V. Blanquet, D. Boichard, D. Rocha, and M. Boussaha. 2017. Identification of copy number variation in French dairy and beef breeds using nextgeneration sequencing. Genet. Sel. Evol. 49:77. https://doi.org/10 $.1186 / \mathrm{s} 12711-017-0352-\mathrm{z}$.

Li, H., B. Handsaker, A. Wysoker, T. Fennell, J. Ruan, N. Homer, G. Marth, G. Abecasis, R. Durbin, and 1000 Genome Project Data Processing Subgroup. 2009. The Sequence Alignment/Map format and SAMtools. Bioinformatics 25:2078-2079. https://doi.org/10 .1093/bioinformatics/btp352.

McKenna, A., M. Hanna, E. Banks, A. Sivachenko, K. Cibulskis, A. Kernytsky, K. Garimella, D. Altshuler, S. Gabriel, M. Daly, and M. A. DePristo. 2010. The Genome Analysis Toolkit: A MapReduce framework for analyzing next-generation DNA sequencing data. Genome Res. 20:1297-1303. https://doi.org/10.1101/gr .107524 .110

Mesbah-Uddin, M., B. Guldbrandtsen, T. Iso-Touru, J. Vilkki, D. J. De Koning, D. Boichard, M. S. Lund, and G. Sahana. 2017. Genome-wide mapping of large deletions and their population-genetic properties in dairy cattle. DNA Res. 25:49-59. https://doi.org/10 $.1093 /$ dnares/dsx037.

Mesbah-Uddin, M., B. Guldbrandtsen, M. S. Lund, and G. Sahana 2018. Genotype call for chromosomal deletions using read-depth from whole genome sequence variants in cattle. Page 662 in Proc. World Congr. Genet. Appl. Livest. Prod., Auckland, New Zealand. AL Rae Centre for Genetics and Breeding, Massey University, Palmerston North, New Zealand. http://www.wcgalp.org/system/ files/proceedings/2018/genotype-call-chromosomal-deletions-using -read-depth-whole-genome-sequence-variants-cattle.pdf.

Mielczarek, M., M. Fraszczak, R. Giannico, G. Minozzi, J. L. Williams, K. Wojdak-Maksymiec, and J. Szyda. 2017. Analysis of copy number variations in Holstein-Friesian cow genomes based on wholegenome sequence data. J. Dairy Sci. 100:5515-5525. https://doi .org/10.3168/jds.2016-11987.

1000 Genomes Project Consortium, A. Auton, L. D. Brooks, R. M. Durbin, E. P. Garrison, H. M. Kang, J. O. Korbel, J. L. Marchini, S. McCarthy, G. A. McVean, and G. R. Abecasis. 2015. A global reference for human genetic variation. Nature 526:68-74. https:// doi.org/10.1038/nature15393.

Pausch, H., I. M. MacLeod, R. Fries, R. Emmerling, P. J. Bowman, H. D. Daetwyler, and M. E. Goddard. 2017. Evaluation of the accuracy of imputed sequence variant genotypes and their utility for causal variant detection in cattle. Genet. Sel. Evol. 49:24. https:// doi.org/10.1186/s12711-017-0301-x.

Quinlan, A. R., and I. M. Hall. 2010. BEDTools: A flexible suite of utilities for comparing genomic features. Bioinformatics 26:841842. https://doi.org/10.1093/bioinformatics/btq033. 
R Core Team. 2014. R: A Language and Environment for Statistical Computing. R Foundation for Statistical Computing, Vienna, Austria.

Sahana, G., T. Iso-Touru, X. Wu, U. S. Nielsen, D. J. de Koning, M. S. Lund, J. Vilkki, and B. Guldbrandtsen. 2016. A 0.5-Mbp deletion on bovine chromosome 23 is a strong candidate for stillbirth in Nordic Red cattle. Genet. Sel. Evol. 48:35. https://doi.org/10 .1186/s12711-016-0215-z.

Schutz, E., C. Wehrhahn, M. Wanjek, R. Bortfeld, W. E. Wemheuer, J. Beck, and B. Brenig. 2016. The Holstein Friesian lethal haplotype 5 (HH5) results from a complete deletion of TBF1M and cholesterol deficiency (CDH) from an ERV-(LTR) insertion into the coding region of APOB. PLoS One 11:e0154602. https://doi .org/10.1371/journal.pone.0154602.

Shin, D. H., H. J. Lee, S. Cho, H. J. Kim, J. Y. Hwang, C. K. Lee, J. Jeong, D. Yoon, and H. Kim. 2014. Deleted copy number variation of Hanwoo and Holstein using next generation sequencing at the population level. BMC Genomics 15:240. https://doi.org/10.1186/ 1471-2164-15-240.

Wu, X., B. Guldbrandtsen, M. S. Lund, and G. Sahana. 2016. Association analysis for feet and legs disorders with whole-genome sequence variants in 3 dairy cattle breeds. J. Dairy Sci. 99:72217231. https://doi.org/10.3168/jds.2015-10705.

Zhang, Q., M. P. L. Calus, M. Bosse, G. Sahana, M. S. Lund, and B. Guldbrandtsen. 2018. Human-mediated introgression of haplotypes in a modern dairy cattle breed. Genetics 209:1305-1317. https://doi.org/10.1534/genetics.118.301143.

Zimin, A. V., A. L. Delcher, L. Florea, D. R. Kelley, M. C. Schatz, D. Puiu, F. Hanrahan, G. Pertea, C. P. Van Tassell, T. S. Sonstegard, G. Marcais, M. Roberts, P. Subramanian, J. A. Yorke, and S. L. Salzberg. 2009. A whole-genome assembly of the domestic cow, Bos taurus. Genome Biol. 10:R42. https://doi.org/10.1186/ gb-2009-10-4-r42.

\section{ORCIDS}

Md Mesbah-Uddin (®) https://orcid.org/0000-0003-1846-0411

Bernt Guldbrandtsen (๑) https://orcid.org/0000-0003-1764-135X

Didier Boichard ๑ https://orcid.org/0000-0003-0361-2961

Goutam Sahana @ https://orcid.org/0000-0001-7608-7577 